\title{
Incidental Discovery of Embryonal Rhabdomyosarcoma on CT Imaging
}

\author{
Esha Jain ${ }^{1}$, Ali Hani Al-Tarbsheh ${ }^{1}$, Jozef Oweis ${ }^{1}$, Hala Abdelwahab² ${ }^{2}$, Boris Shkolnik ${ }^{3}$ \\ ${ }^{1}$ Department of Medicine, Albany Medical Center, Albany, New York, USA \\ ${ }^{2}$ Department of Pathology, Albany Medical Center, Albany, New York, USA \\ ${ }^{3}$ Department of Medicine, Division of Pulmonary and Critical Care Medicine, Albany Medical Center, Albany, New York, USA
}

Received: 23/10/2021

Accepted: 08/11/2021

Published: $24 / 11 / 2021$

How to cite this article: Jain E, Al-Tarbsheh AH, Oweis J, Abdelwahab H, Shkolnik B. Incidental discovevry of embryonal rhabdomyosarcoma on CT imaging. EJCRIM 2021;8: doi:10.12890/2021_002834.

Conflicts of Interests: The authors declare there are no competing interests.

Acknowledgements: We thank Divya B Dasani, BA and Amanda N Awad, BS, for help writing and editing the manuscript.

This article is licensed under a Commons Attribution Non-Commercial 4.0 License

\section{ABSTRACT}

Rhabdomyosarcoma is an uncommon soft tissue sarcoma that rarely presents in adults. Clinical presentation is dependent on site and size. We present the case of a woman who presented with acute-onset dyspnoea and whose pathology report confirmed embryonal rhabdomyosarcoma (ERMS) seen as an incidental finding on chest computed tomography. We also describe the clinical, laboratory and radiological work-up conducted to diagnose and manage ERMS in the critical care setting.

\section{LEARNING POINTS}

- Rhabdomyosarcoma is a rare malignancy with a poor prognosis in adults compared with children, especially if it presents in an unfavourable primary site and has an unfavourable histological diagnosis.

- Immunohistochemical diagnosis remains the gold standard for embryonal rhabdomyosarcoma diagnosis and differentiation from similar malignancies on initial imaging studies.

- Management of adult rhabdomyosarcoma is usually multimodal with surgical resection and a combination of chemo and radiotherapy.

\section{KEYWORDS}

Rhabdomyosarcoma, embryonal rhabdomyosarcoma, immunohistochemistry

\section{INTRODUCTION}

Rhabdomyosarcoma (RMS) is a rare soft tissue sarcoma that typically presents in children and adolescents ${ }^{[1]}$. It is divided into four histological subtypes: embryonal, alveolar, anaplastic and sclerosing ${ }^{[2]}$. In adults, fewer than $1 \%$ of all solid malignant cancers are sarcomas, and less than 2-5\% of adult sarcomas are RMS ${ }^{[3]}$. Embryonal rhabdomyosarcoma (ERMS) accounts for the majority of cases. ERMS in adults can occur at any site, but most commonly arises from the genitourinary or the head and neck region ${ }^{[4]}$. We present the case of a woman who presented with shortness of breath and was found to have mediastinal ERMS.

\section{CASE DESCRIPTION}

A 28-year-old Caucasian woman presented with a 2-month history of shortness of breath, exertional dyspnoea, and productive cough. She endorsed profuse sweating along with a 10-pound weight loss over several weeks prior to presentation. She did not have any fever, abdominal pain, chest pain or haemoptysis. Her medical history was not significant for any medical problems.

Blood pressure on admission was 122/59 $\mathrm{mmHg}$ measured on both arms, heart rate was 104/min, respiratory rate was 18/min, and temperature was $37.1^{\circ} \mathrm{C}$. Her oxygen saturation was $97 \%$ on room air. The patient had a plethoric facial appearance. Physical examination 
revealed depressed lung sounds over the right middle lobe. The remaining lung areas were clear on auscultation, and the abdomen was soft and non-tender.

Laboratory tests showed an elevated international normalized ratio of 1.2 (0.83-1.14). A complete blood count disclosed a haemoglobin of $9.7 \mathrm{~g} / \mathrm{dl}(13.6-16.7 \mathrm{~g} / \mathrm{dl})$, a haematocrit of $29.2 \%$ (40-49\%), a white blood cell count of 14,000/mm3 (4-9/.mm $\left.{ }^{3}\right)$, and a platelet count of $197,000 / \mathrm{mm}^{3}\left(130,000-350,000 / \mathrm{mm}^{3}\right)$.

Emergent imaging was obtained due to concern for superior vena cava syndrome.

CT of the chest on admission revealed a large ill-defined mediastinal and right lung mass with mediastinal shift to the left (Fig. 1), and a large, right pleural effusion (Fig. 2). An echocardiogram of the heart to investigate cardiopulmonary aetiology showed a small to moderate pericardial effusion without tamponade and LVEF $>70 \%$.

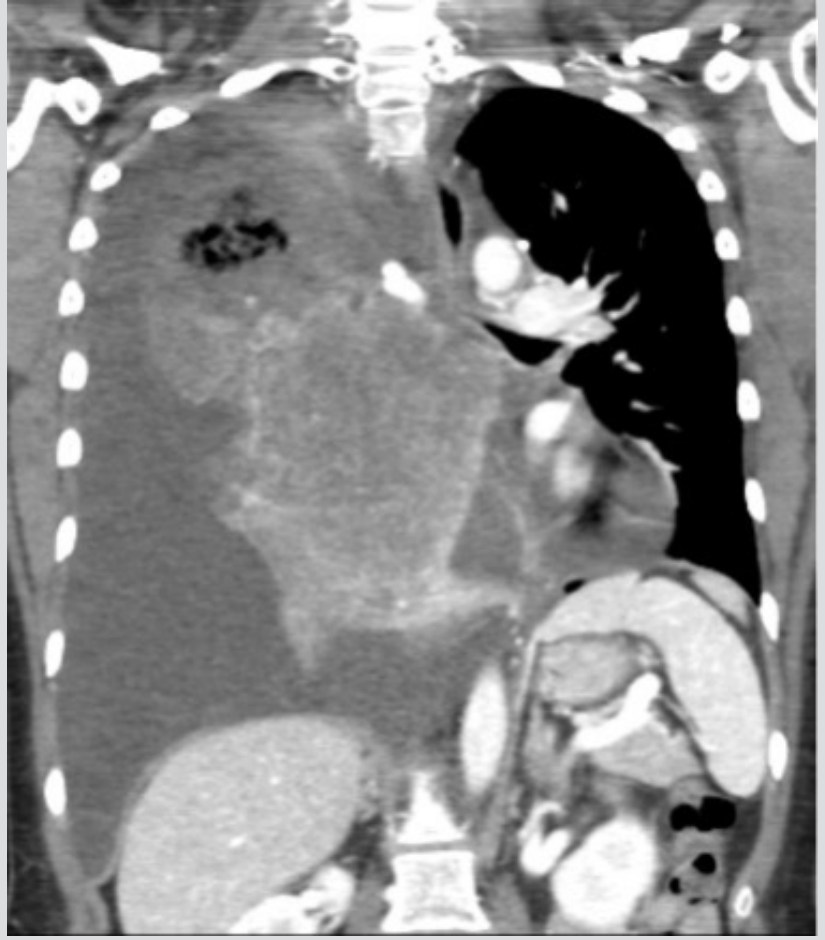

Figure 1. CT chest radiograph on admission showing a large right lung mass associated with mass effect on the heart and mediastinal shift to the left

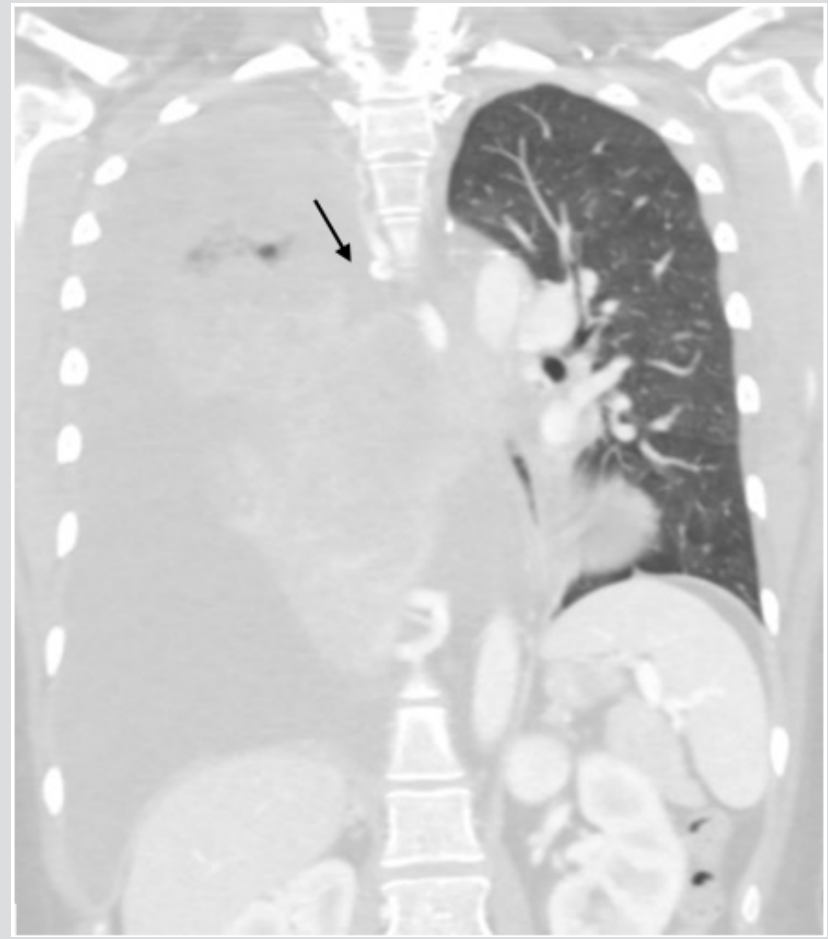

Figure 2. CT chest radiograph on admission showing a large perihilar right lung mass compressing the right mainstem bronchus, resulting in near complete atelectasis of the right lung. There is a large, right pleural effusion (arrow)[

An ultrasound-guided right-sided thoracentesis was conducted to investigate pulmonary malignancy. Pleural fluid disclosed a malignant round cell tumour consistent with RMS. Subsequently, the patient underwent a right-sided bronchoscopy with endobronchial ultrasound (EBUS) for specimen retrieval. The pathology report of the bronchial specimen confirmed ERMS.

Two weeks after admission, CT chest radiography showed resolution of the pleural effusion and partial re-expansion of the right lung. However, there was a new right pneumothorax, so a chest tube was placed (Fig. 3). Two months after admission, CT chest radiography showed resolving airspace with new pleural thickening on the right hemithorax with a new air/fluid collection (Fig. 4). Three months after admission, the patient exhibited increased ground-glass opacity and consolidation in the right lung with new ground-glass opacity throughout the left lung. A second collection was also noted on the CT chest radiograph (Fig. 5). The patient was started on linezolid and piperacillin-tazobactam and later discharged on amoxicillin-clavulanate for possible infectious aetiology. The decision was made to institute palliative chemotherapy. Unfortunately, the patient's shortness of breath and fatigue continued to worsen. She was sent to our centre for further evaluation but decided to withdraw from treatment and pursue hospice care. 


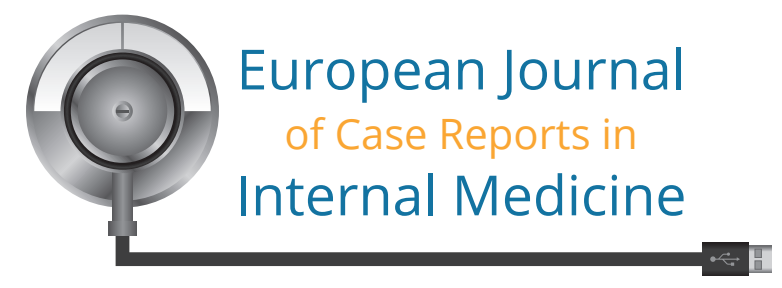

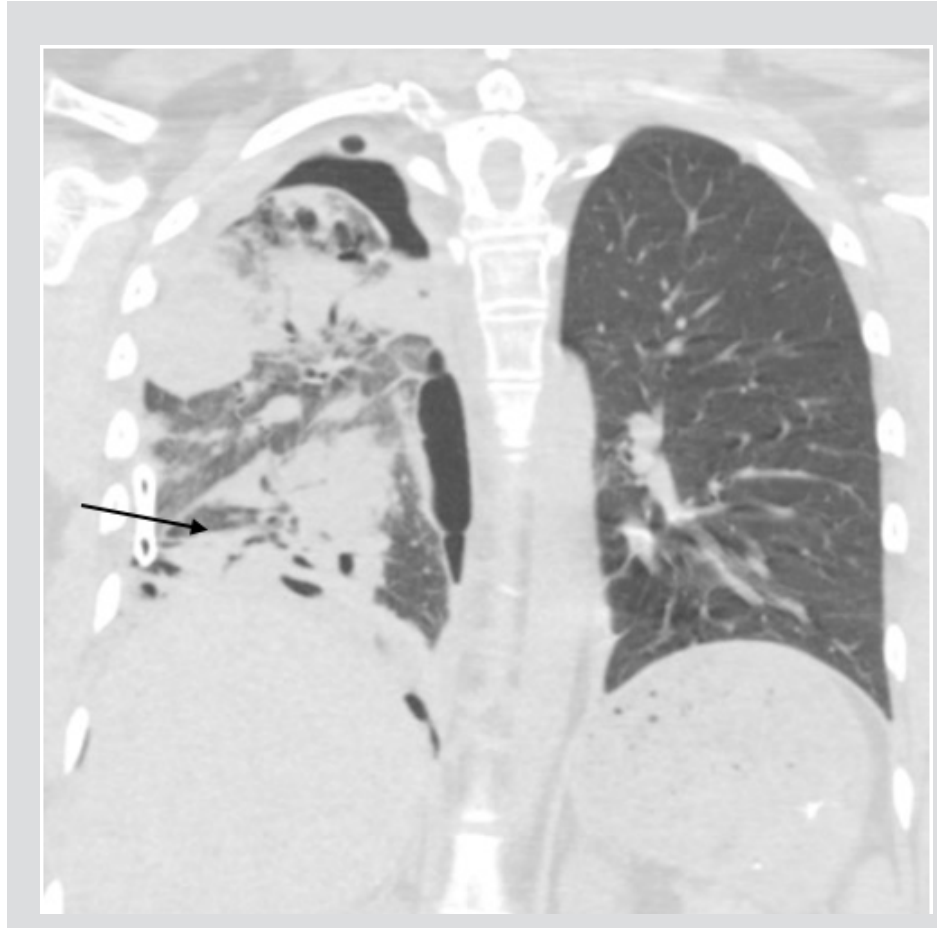

Figure 3. Chest CT radiograph showing resolution of the pleural effusion and partial reexpansion of the right lung, which shows diffuse ground-glass attenuation and scattered nodular consolidation with peribronchovascular thickening with traction bronchiectasis in the lower lobe. A new right pneumothorax with placement of a chest tube can be seen near the right lung base (arrow)

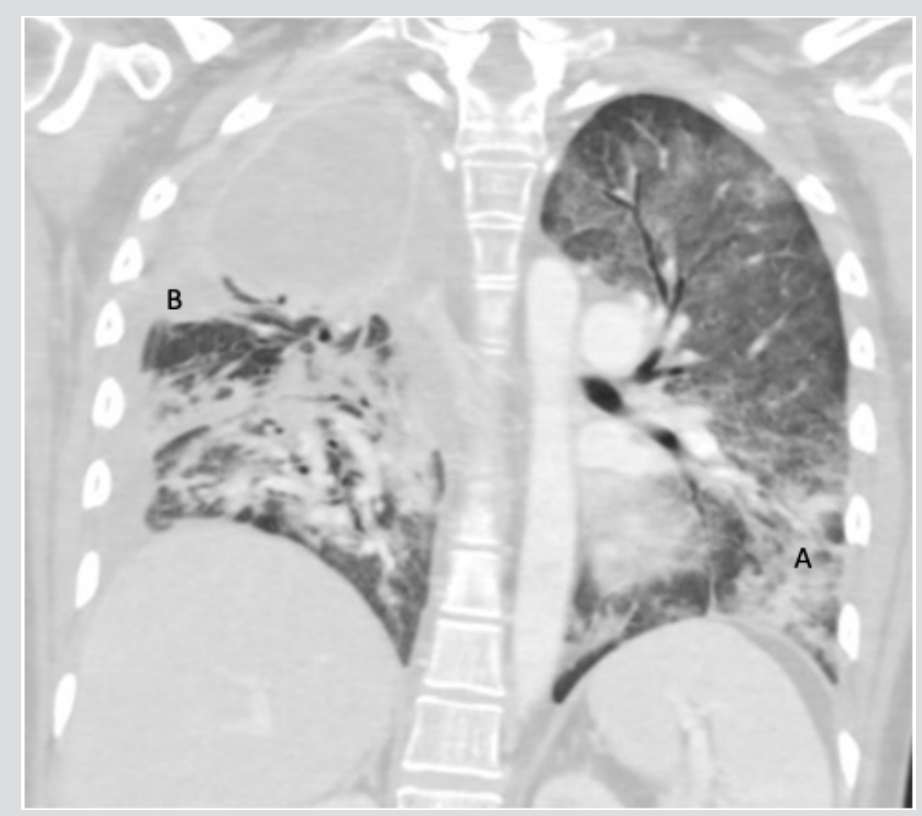

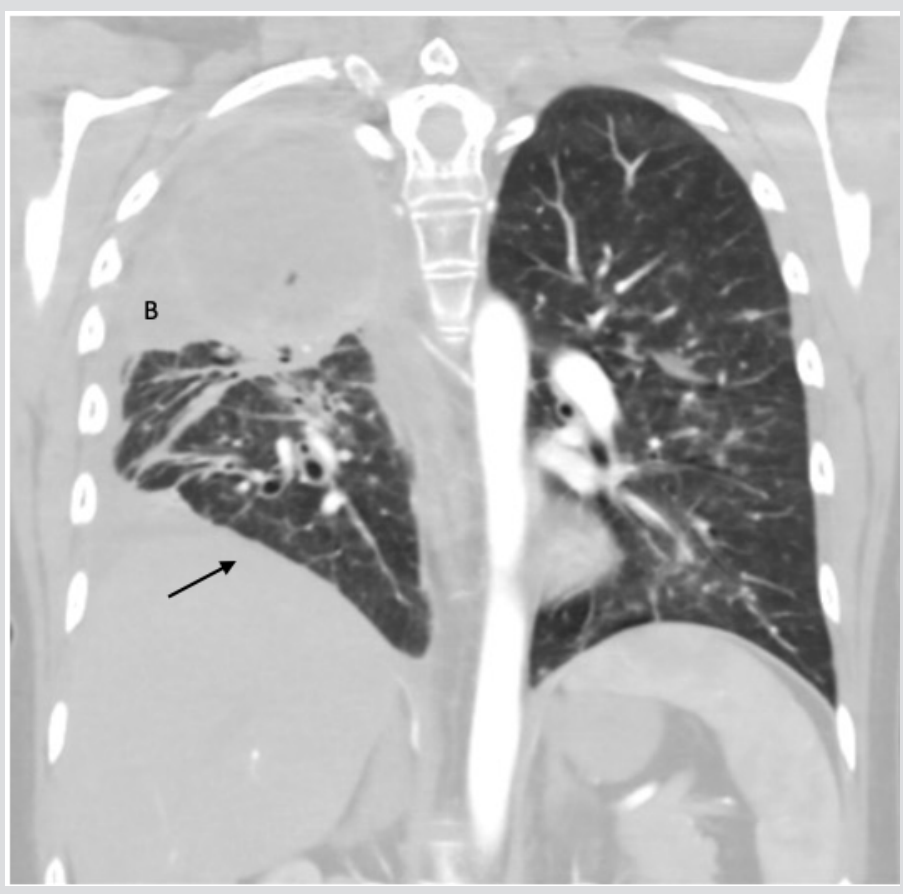

Figure 4. CT chest radiograph several months after admission showing resolving airspace disease (B). New pleural thickening throughout the right hemithorax with a new apical loculated air/fluid collection can be seen (arrow)
Figure 5. CT chest radiograph showing increased ground-glass opacity and consolidation in the right lung with new appearance of ground-glass opacity throughout the left lung (A). A second, smaller collection is now noted abutting the inferior aspect of apical empyema (B)

\section{DISCUSSION}

Embryonal rhabdomyosarcoma is one of the most common soft tissue cancers diagnosed in children and adolescents ${ }^{[5,6]}$. However, ERMS in adults is extremely rare and primarily arises from the head, neck and extremities ${ }^{[5,7]}$. Less commonly, RMS develops in striated muscle, including in the lungs ${ }^{[8]}$. The literature on EMRS in adults is limited and there are few studies regarding management of these patients. 
However, research has continued on ERMS in adults despite its rarity. Lloyd and colleagues describe a series of 54 cases of ERMS in adults during a 28-year period. They compared recurrence rate, metastasis and death from disease in patients treated in two different time periods. Patients in the later period had improved survival, perhaps due to better understanding and better histological diagnosis of the disease ${ }^{[6]}$. There are few case reports regarding the initial clinical presentation of adult patients with ERMS. Linton and colleagues have described the importance of the clinical presentation of ERMS based on its primary site. Their patient presented with worsening dyspnoea with existing stridor and hoarseness ${ }^{[4]}$. In the current clinical report, our patient complained of worsening shortness of breath, dyspnoea on exertion, and a productive cough. RMS causes local symptoms related to mass lesions, infiltration and destruction of adjacent tissues, which contribute to its diverse and non-specific clinical manifestations, resulting in frequent misdiagnosis ${ }^{[5,9]}$.

Only three other case reports have described the initial diagnosis of ERMS through pleural fluid analysis and subsequent immunohistochemistry (IHC). Thompson and colleagues reported a 31-year-old Caucasian man who presented with ERMS of the testis who underwent radical left orchiectomy. One year after surgical resection, the patient presented with pleural effusion and died 6 months after the diagnosis of malignant pleural effusion ${ }^{[10]}$. Cohen et al. reported a case of ERMS in an 11-year old boy investigated for mild chest pain after trauma, with radiographic evidence of a massive pleural effusion. Further cytological analysis of the pleural fluid demonstrated malignant small undifferentiated cells, which were confirmed to be rhabdomyoblastic by positive immunostaining ${ }^{[11]}$. In our case, the patient initially presented with a primary lung mass and was diagnosed through pleural fluid analysis and IHC staining, which in another case had previously proved to be very useful for accurate diagnosis ${ }^{[11]}$.

Primary pulmonary RMS is extremely rare and typically presents as a parenchymal mass ${ }^{[12]}$. Ji and colleague have described this in a case report discussing the only histologically confirmed case of RMS between 1995 and 2021 in western China ${ }^{[5]}$. CT radiography revealed a soft tissue mass in their patient's upper left lung. IHC staining showed tumour cells positive for desmin and partially positive for myogenin. In the current clinical case, our patient was found to have a pleural effusion and a mediastinal mass on X-ray and CT radiography. IHC staining demonstrated the tumour cells were positive for myogenin, desmin, vimentin and TLE-1. There was weak positive staining for synaptophysin and ALK-1. Although definitive subtyping was complicated, it was likely consistent with ERMS. Expression of these muscle-specific proteins helps to confirm the diagnosis in unusual locations [8]. Furthermore, diagnosis of this malignancy depends mainly on a combination of pathological, IHC and molecular biological findings. Further studies on the histological features of RMS are required (Fig. 6) ${ }^{[5]}$.

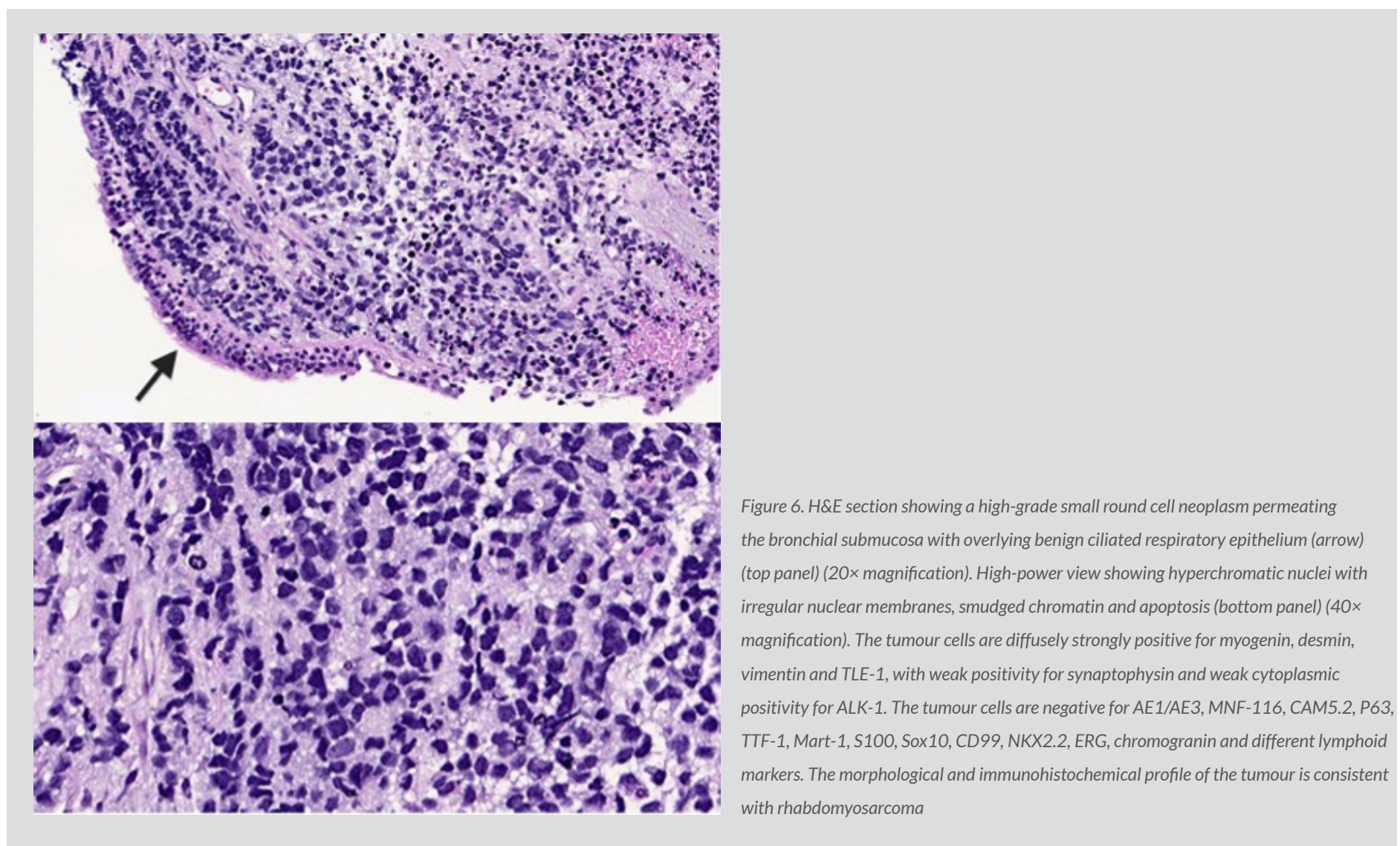


ERMS is an unusual soft tissue sarcoma that is typically diagnosed in children but is rare in adult patients and associated with a poor prognosis ${ }^{[13]}$. Proper radiographic imaging and IHC staining are essential, as demonstrated by the current case. It is a rare malignancy that can present with non-specific symptoms. This case report highlights the importance of thorough clinical work-up in order to better diagnose and manage patients.

\section{REFERENCES}

1. Dasgupta R, Fuchs J, Rodeberg D. Rhabdomyosarcoma. Semin Pediatr Surg 2016;25(5):276-283.

2. Clay M. Embryonal rhabdomyosarcoma. PathologyOutlines.com website. https://www.pathologyoutlines.com/topic/softtissueembryonalrhabdo.html. Accessed November 8th, 2021.

3. Taza F, Kanwal A, Zulty M, Mustafa S. High-grade pleomorphic rhabdomyosarcoma in a 60-year-old male: a case report and review of the literature. J Community Hosp Intern Med Perspect 2020;10(3):287-289.

4. Linton S, Stapleton E, Penney S, Sharma M-P. Embryonal rhabdomyosarcoma masquerading as a left vocal cord granuloma. BMJ Case Rep 2020;13(10):e237025.

5. Ji G-y, Mao H. Primary pulmonary rhabdomyosarcoma in an adult: a case report and review of the literature. J Zhejiang Univ Sci B 2013;14(9):859-865.

6. Lloyd RV, Hajdu SI, Knapper WH. Embryonal rhabdomyosarcoma in adults. Cancer 1983;51(3):557-565.

7. Mysorekar VV, Harish K, Kilara N, Subramanian M, Giridhar AG. Embryonal rhabdomyosarcoma of the chest wall: a case report and review of the literature. Indian J Pathol Microbiol 2008;51(2):274-276.

8. Balaji R, Kumar P, Garg I, Das K. Primary pulmonary rhabdomyosarcoma in a child. J Indian Assoc Pediatr Surg 2017;22(1):57-59.

9. Parham DM, Ellison DA. Rhabdomyosarcomas in adults and children: an update. Arch Pathol Lab Med 2006;130(10):1454-1465.

10. Thompson KS, Jensen JD, Bhoopalam N, Reyes CV. Pleural effusion cytology of embryonal rhabdomyosarcoma. Diagn Cytopathol 1997;16(3):270-273.

11. Cohen I, Loberant N, King E, Herskovits M, Sweed Y, Jerushalmi J. Rhabdomyosarcoma in a child with massive pleural effusion: cytological diagnosis from pleural fluid. Diagn Cytopathol 1999;21(2):125-128.

12. Gupta A, Sharma MC, Kochupillai V, Kichendasse G, Gupta A, Atri S, et al. Primary pulmonary rhabdomyosarcoma in adults: case report and review of literature. Clin Lung Cancer 2007;8(6):389-391.

13. Hardaway CA, Graham BS, Barnette DJ, Feldman BD. Embryonal rhabdomyosarcoma presenting in an adult: a case report and discussion of immunohistochemical staining. Am J Dermatopathol 2003;25(1):45-52. 